\title{
AXIOMATIC DEFINITIONS OF PERFECTLY SEPARABLE METRIC SPACES*
}

\section{BY GARRETT BIRKHOFF}

1. Introduction. Among topological spaces, perfectly separable metric spaces (abbreviated PSM-spaces) play a major $\dagger$ role; therefore their axiomatic definition is a problem of peculiar interest. We shall discuss axiomatic definitions of PSM-spaces.

A topological space is $\ddagger$ a class $\subseteq$ of "points" $x$, and an operation $K$ assigning to any set $S$ of points of $\mathfrak{\Im}$, a "derived" set $K(S)$. In practice, $K$ is defined in one of three ways:

I. By ascribing to every point $x$ of $\mathfrak{S}$ a family of "neighborhoods of $x$," denoted by $V_{x}$ with variable superscript. Here $K(S) \supset x$, if and only if no $\left(V_{x}-x\right) \cdot S$ is vacuous.

II. By ascribing to every ordered pair $(x, y)$ of points of $\mathfrak{S}$, a number $\overline{x y} \cdot \overline{x y}>0$ if $x \neq y ; \overline{x x}=0$. Here $K(S) \supset x$, if and only if g.l.b. $s_{\supset x \neq y} \overline{x y}=0$.

III. By ascribing to every point $x$ of $\subseteq$ a family of infinite sequences $\left\{x_{k}\right\}$ of distinct points of $\subseteq$, which "converge to $x$ " (abbreviated $x_{k} \rightarrow x$ ). Here $K(S) \supset x$, if and only if we can find a sequence in $S$ converging to $x$.

Spaces defined as in I are known as spaces $(V)$; we shall call spaces defined as in II spaces $(Q)$, and those defined as in III, spaces $(\Lambda)$.

A system of neighborhood axioms, such that any space $(V)$ satisfying it is a PSM-space, has been deduced by HausdorffUrysohn-Tychonoff. $\S$ With slight modifications, the axioms are satisfied by every representation of a PSM-space as a space $(V)$. It is therefore futile to attempt to weaken them except as regards interdependence.

* Adapted from a Harvard Undergraduate Honors Thesis.

$\dagger$ For they are precisely the class used in function theory. See Mathematische Annalen, vol. 92 (1924), p. 302.

$\ddagger$ Fréchet, Les Espaces Abstraits, 1928, p. 167.

$\S$ See Hausdorff's Grundzüge der Mengenlehre, 1914, Chaps. 7-8; Urysohn, Zum Metrisationsproblem, Mathematische Annalen, vol. 94 (1925), p. 309; Tychonoff, Über ein Satz, etc., Mathematische Annalen, vol. 95 (1925), p. 139. 
Systems of distance axioms, all spaces $(Q)$ that satisfy which are metric spaces, have been deduced by Chittenden-NiemytskiWilson.* We shall proceed by another line to find a system of distance axioms, effectively weaker under the additional hypothesis of perfect separability - satisfied in fact by every representation of a PSM-space as a space $(Q)$. No system of convergence axioms, all spaces $(\Lambda)$ that satisfy which are PSM-spaces, has, I believe, been set up hitherto. We shall construct such a system, showing in what sense it is necessary as well as sufficient.

2. Spaces $(\Lambda)$ and Spaces $(Q)$. The class of spaces $(\Lambda)$ is quite evidently topologically equivalent $\dagger$ (abbreviated TE) to the class of spaces $(V)$ in which, if $K(S) \supset x, S$ contains an enumerable subset $S^{\prime}$ such that $K\left(S^{\prime}\right) \supset x$. Suppose that in addition we can so choose $S^{\prime}$ that $\left\{y_{k}\right\} \subset S^{\prime}$ implies $K\left(\left\{y_{k}\right\}\right)$ $\supset x$. We can make the class of such sets $S^{\prime}$ our fundamental class of convergent sequences. We then have a space $(\Lambda)$ in which

(A) If $x_{k} \rightarrow x$, and $\left\{y_{k}\right\} \subset\left\{x_{k}\right\}$, then $y_{k} \rightarrow x$. Conversely if, given $\left\{y_{k}\right\} \subset\left\{x_{k}\right\}$, we can so choose $\left\{z_{k}\right\} \subset\left\{y_{k}\right\}$ that $z_{k} \rightarrow x$, then $x_{k} \rightarrow x$.

THeOREM 1. Let $(\Im, K)$ be a space $(\Lambda)$ satisfying

(B) If $x_{k} \rightarrow x$, and $\left\{y_{k}\right\} \subset\left\{x_{k}\right\}$, then we can so choose $\left\{z_{k}\right\} \subset\left\{y_{k}\right\}$ that $z_{k} \rightarrow x$.

Then $(\mathfrak{S}, K)$ has a TE representation as a space $(\Lambda)$ satisfying (A).

For add to the primitive families of convergent sequences of $(\subseteq, K)$, all sequences satisfying the second half of (A). Then $K$ will be unchanged, yet (A) will be satisfied by the augmented families of convergent sequences. It is noteworthy that any further augmentation either contradicts (B) or changes $K$.

A space is called locally separable if and only if it is TE to a space $(V)$ in which the family of neighborhoods ascribed to any $x$ can be enumerated: $V_{x}^{1}, V_{x}^{2}, V_{x}^{3}, \ldots$. Every space $(Q)$ is locally separable; let $V_{x}^{n} \supset y$ if and only if $\overline{x y}<1 / n$. Every space

* For latest results see Niemytski's $O n$ the third axiom of metric space, Transactions of this Society, vol. 29 (1927), p. 666; also W. A. Wilson's two articles in the American Journal, vol. 53 (1931).

† Two topological spaces are called "topologically equivalent" if and only if a $(1,1)$-correspondence which preserves $K$ can be established between their points. 
$(\Lambda)$ is* a space $(V)$, but not necessarily a locally separable one. Let $(\Im, K)$ be a locally separable space $(\Lambda)$, and define a pseudo-distance $\tilde{x y}$ as follows: $\tilde{x x}=0$; and $\tilde{x y}=1 / n[x \neq y]$, if $V_{x}^{n}$ is the first $V_{x}$ in order of enumeration not $\dagger$ containing $y$. If g.l.b. $s \supset_{y \neq x} \tilde{x y}=0$, then plainly $K(S) \supset x$; i.e., given $n, y \subset S$ exists contained in at least the first $n$ neighborhood $V_{x}^{k}$. Suppose $K(S) \supset x$, yet g.l.b. $s \supset y \neq x \tilde{x y}>0$. Hence $S \supset x_{k} \rightarrow x$, yetg.1.b. $\tilde{x} x_{k}>0$; therefore that some $V_{x}^{n}$ excludes an infinity of the $x_{k}$. Extracting the subsequence not in $V_{x}^{n}$, we obtain an exception to (A).

Conversely, in a space $(Q)$, let us write $x_{k} \rightarrow x$ if and only if $\overline{x x}_{k} \rightarrow 0$. It follows from the theory of limits, that $K(S) \supset y$ if and only if an infinite sequence $\left\{x_{k}\right\}$ exists in $S$, satisfying $x_{k} \rightarrow y$. Moreover the pseudo-convergences just defined satisfy (A). We may therefore state the following theorem.

TheOREM 2. The class of locally separable spaces $(\Lambda)$ satisfying (A) is TE to the class of spaces $(Q)$. Moreover $\overline{x x}_{k} \rightarrow 0$ is equivalent to $x_{k} \rightarrow x$.

3. Locally Separable Hausdorff Spaces. Consider ( $(\mathfrak{S}, K)$, any space $(Q)$ satisfying the two conditions

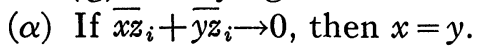

( $\beta$ ) If $\overline{x x}_{i} \rightarrow 0$, and $\overline{x_{i} x_{j}{ }^{i}} \rightarrow 0$ identically in $i$, then $N(i)$ exists so large that $j(i)>N(i)$ implies $\overline{x x}_{j(i)} \rightarrow 0$.

LEMma. $K\{K(S)\} \subset K(S)$ for any set $S$ in $(\Im, K)$.

For suppose $x \subset K\{K(S)\}$; we can evidently so choose $x_{i} \subset K(S)$ that $\overline{x x}_{i} \rightarrow 0$. But since $x_{i} \subset K(S)$, we can so choose $x_{j}^{i} \neq x$ in $S$ that $\overline{x_{i} x_{j}} \rightarrow 0$, irrespective of $i$. Hence by $(\beta)$, g.1.b. $s \supset x_{i}^{i} \neq \bar{x}^{x} \bar{x}_{j}^{i}=0$, and $x \subset K(S)$.

Let us define $V_{x}^{n}[x \subset \subseteq ; n=1,2,3, \ldots]$ as the set of all points $y$ which satisfy $\overline{x y}>1 / n$; let $W_{x}^{n}=V_{x}^{n}-K\left(\Im-V_{x}^{n}\right)$. Then $V_{x}^{n} \supset x$, but $x$ is not $\subset K\left(\Im-V_{x}^{n}\right)$, so that the following statement will be true.

(a) To every $x$ corresponds a $W_{x}^{n}$, and every $W_{x}^{n}$ contains $x$. Moreover the theory of limits shows us that in any space $(Q)$, $K(S+T)=K(S)+K(T)$. It follows by this and the Lemma that

* Since $S \supset S^{\prime}$ implies $K(S) \supset K\left(S^{\prime}\right)$; see Les Espaces Abstraits, p. 179.

$\dagger$ If $x \neq y$, some $V_{x}^{n} \supset y$; otherwise $K(y) \supset x$, and since $y$ contains no infinite sequence, this is impossible. 


$$
\begin{aligned}
K\left(\mathfrak{S}-W_{x^{i}}\right) & =K\left(\mathfrak{S}-V_{x^{n}}\right)+K\left\{K\left(\mathfrak{S}-V_{x^{n}}\right)\right\} \\
& =K\left(\mathfrak{S}-V_{x^{n}}\right) \subset \mathfrak{S}-W_{x^{n}} .
\end{aligned}
$$

Now suppose that some $\left(W_{x}^{n}-x\right) \cdot S$ is vacuous, so that $\left(\Im-W_{x}^{n}\right)+x \supset S$. It follows that $K\left(\Im-W_{x}^{n}\right)+K(x) \supset K(S)$; hence, using $(\phi)$ and the fact that $K(x)$ is null, we see that (ऽ$\left.W_{x}^{n}\right) \supset K(S)$, and, by (a), $K(S)$ is not $\supset x$. Conversely, if no $\left(W_{x}^{n}-x\right) \cdot S$ is vacuous, certainly no $\left(V_{x}^{n}-x\right) \cdot S$ is vacuous, and $K(S) \supset x$. That is, the $W_{x}^{n}$ are neighborhoods of $x$ in the sense of $\mathrm{I}$.

(b) Let $W_{x}^{i}$ and $W_{x}^{j}$ be given. Then $W_{x}^{i+j} \subset W_{x}^{i} \cdot W_{x}^{j}$ exists.

(c) If $y \subset W_{x}^{n}$, then $y$ is not $\subset\left(\varsigma-W_{x}^{n}\right)$, whence by $(\phi)$, we have $y$ is not $\subset K\left(\subseteq-W_{x}^{n}\right)$. Therefore $W_{y}^{n}$ exists, making $W_{y}^{n} \cdot\left(\Im-W_{x}^{n}\right)$ null, and hence contained in $W_{x}^{n}$.

(d) Let $x \neq y$ be given. Then $W_{x}^{n}$ and $W_{y}^{n}$ exist without common point; otherwise $z_{n} \subset W_{x}^{n}, W_{y}^{n}$ exists for every $n$, and $\overline{x z}_{n}+\overline{y z}_{n} \rightarrow 0$, contradicting $(\alpha)$.

Conversely, setting up a pseudo-distance just as in the case of locally separable spaces $(\Lambda)$, we obtain a TE space $(Q)$ from any space $(V)$ which is locally separable and satisfies (a) and (b). Further, we can obtain $(\alpha)$ from (d) and $(\beta)$ from (c). Therefore we may state the following result.

THEOREM 3. The class of spaces $(Q)$ satisfying $(\alpha)$ and $(\beta)$ is $\mathrm{TE}$ to the class of locally separable Hausdorff spaces.

4. Two Further Conditions. A topological space is said to be regular* if and only if to every point $x$ and every closed set $S$ not containing $x$ correspond disjoint open sets $U \supset x$ and $V \supset S$. Suppose $(\widetilde{S}, K)$ is a space $(Q)$ satisfying $(\alpha)$ and $(\beta)$ but not regular, and let $x$ and $S$ defined as above be contained in no two disjoint open sets. Let finally $W$ be defined as in $\$ 3$.

Every $W_{x}^{i}$ must have either a point or a limit point lying in $S$; otherwise we could enclose each point of $S$ in an open set disjoint to $W_{x}^{i}$, and the sum of these open sets would be an open set containing $S$ and disjoint to $W_{x}^{i} \supset x$. Since $K(S)$ is not $\supset x, W_{x}^{i} \cdot S$ is vacuous for $i>M(S, x)$, and to $i>M(S, x)$ correspond $x_{j}^{i} \subset W_{x}^{i}$ such that $x_{j}^{i} \rightarrow x_{i} \subset S$. But since $x$ is not $\supset S \supset$ $K(S)$, g.l.b. $S_{S} x_{i}^{i} \overline{x x_{i}^{i}}>0$. That is, if we set $N(i)=0$, there exists an exception to this statement:

* E. W. Chittenden, this Bulletin, vol. 33 (1927), p. 20. The term is due to Alexandroff and Urysohn, Mathematische Annalen, vol. 92 (1924), p. 263. 
$\left(\beta^{\prime}\right)$ If $N(i)$ exists so large that $j(i)>N(i)$ implies $\overline{x x}_{j(i)}^{i} \rightarrow 0$, and $\bar{x}_{i} x_{j}^{i} \rightarrow 0$ identically in $i$, then $\overline{x x}_{i} \rightarrow 0$.

We have therefore proved the following fact.

TheOREM 4. Every space $(Q)$ satisfying $(\alpha),(\beta)$, and $\left(\beta^{\prime}\right)$ is TE to a regular locally separable Hausdorff space.*

We shall call a space perfectly separable if and only if we have:

( $\gamma$ ) There exist open sets $N_{1}, N_{2}, N_{3}, \cdots$, of which any open set is the sum of a subclass.

THEOREM 5. If a Hausdorff space satisfies $(\gamma)$, it is perfectly separable in the sense of Hausdorff, that is, we can take the $N_{i}$ for neighborhoods; and conversely.

Let $N_{i}$ be a neighborhood of $x$ if and only if $N_{i} \supset x$, and observe (1) that since each $N_{i}$ is open, it contains at least one neighborhood of every point contained in itself; (2) that since by (c) every $V_{x}$ is open, $V_{x}=\sum N_{i}$ summed over a suitable range of $i$,-whence since in addition by (A) $V_{x} \supset x$, it follows that $V_{x} \supset N_{i} \supset x$ is satisfied by at least one $N_{i}$. It results from (1) and (2) that the $N_{i}$ define $K$ correctly, while (A) - (D) can be easily deduced from (1), (2), and the fact that the $N_{i}$ are open sets containing the points of which they are neighborhoods. To prove the converse, merely set the $V_{x}$ as $N_{i}$.

Theorem 6. If the derived sets of a space $(\Lambda)$ are closed, and it satisfies $(\gamma)$, it is locally separable.

For in this case the interior points of neighborhoods of a point form an equivalent $\dagger$ system of neighborhoods and are open; from this point we can proceed as in the proof of the first part of Theorem 5 .

If we note that $\overline{x x}_{i} \rightarrow 0$ means simply that $k_{i}<k_{i+1}$ implies $K\left(\sum_{1}^{\infty} x_{k_{i}}\right) \supset x$, we see immediately that conditions $(\alpha)-(\gamma)$ are topologically invariant. Likewise, since under any metric $\ddagger$ representation $\overline{x z_{i}}+\overline{y z_{i}} \geqq \overline{x y}$, while we can so choose $N(i)$ that $\bar{x}_{i} x_{j}^{i}<1 / i$, whence $\left|\overline{x x}_{i}-\overline{x x}_{j}{ }^{i}\right|<1 / i$, for $j>N(i)$, we see that $(\alpha)-(\gamma)$ hold under the metric representation of a PSM-space.

* The converse is also true.

$\dagger$ Les Espaces Abstraits, p. 173.

$\ddagger$ A metric representation is a representation as a space $(Q)$ in which a. $\overline{x y}=\overline{y x}, \mathrm{~b}, \overline{x y}+\overline{y z} \geqq \overline{x z}$. 
Hence $(\alpha)-(\gamma)$ hold under any representation of a PSM-space as a space $(Q)$. However, Tychonoff $\dagger$ has proved that a necessary and sufficient condition that a perfectly separable Hausdorff space be metric is that it be regular. Applying Theorems 4 and 5 , we may therefore state the following conclusion.

Theorem 7. A necessary and sufficient condition that a space $(Q)$ be a PSM-space is that $(\alpha),(\beta),\left(\beta^{\prime}\right)$, and $(\gamma)$ hold.

5. Conclusions. Inasmuch as every space $(Q)$ is locally separable, and $\boldsymbol{\aleph}^{2}=\boldsymbol{\aleph}$, it might be conjectured that $(\gamma)$ was replaceable by the following neater condition:

$\left(\gamma^{\prime}\right) \quad \Gamma=x_{1}+x_{2}+x_{3}+\cdots$ exists in $\subseteq$, and $K(\Gamma)=\mathfrak{S}$.

THEOREM 8. There exists a space $(Q)$ which is not a PSM-space, yet which satisfies $(\alpha),(\beta),\left(\beta^{\prime}\right)$, and $\left(\gamma^{\prime}\right)$.

For let $\subseteq$ consist of all points $x[0<x<1]$, and let $\overline{x y}=(x-y)$ or 1 , according as $x \geqq y$ or $x<y$. That $(\Im, K)$ satisfies the hypotheses is fairly obvious; the only difficulty is to show that it is not perfectly separable. To show this, remark that every $V_{x_{0}}$ contains a set $x_{0}>x>x_{0}-\epsilon$, and some $V_{x_{0}}^{*}$ excludes the entire set $x<x_{0}$ as well. Now $V_{x}{ }^{*}=V_{y}{ }^{*}$ only if $x=y$. Hence the neighborhoods are certainly not enumerable.

Let us disprove in the same way a second conceivable conjecture. Let $\subseteq$ consist of two classes of points, points $p_{k}$ and points $q_{k}[0=k=1]$. Let $\overline{q_{i} q_{j}}=\overline{q_{i} p_{j}}=\overline{p_{k} q_{k}}=\widehat{q_{k} p_{k}}=1$, and $\overline{p_{i} p_{j}}=\overline{p_{i} q_{j}}$ $=|i-j|,[i \neq j]$. It is easy to verify, using Theorem 4 , that the space is a compact regular locally separable Hausdorff space. It is not perfectly separable, as it contains more than countable isolated points. Therefore it is not metric; every compact metric space isł perfectly separable. Hence we have the following fact.

THEOREM 9. There exists a compact regular locally separable Hausdorff space which is not metric.

By simple ad hoc constructions we can show the independence of $(\alpha),(\beta)+\left(\beta^{\prime}\right)$, and $(\gamma)$ as conditions on a space $(Q)$; the last

$\dagger$ Mathematische Annalen, vol. 95 (1925), p. 139.

‡ See Urysohn, Zum Metrisationsproblem, Mathematische Annalen, vol. 94 (1925), p. 309. 
is incidentally proved by Theorem 8 . From this and Theorem 7 we have the following result.

THEOREM 10. The following distance axioms determine which spaces $(Q)$ are PSM-spaces:

( $\alpha$ ) If $\overline{x z}_{i}+\overline{y z}_{i} \rightarrow 0$, then $x=y$.

$\left(\beta^{\prime \prime}\right)$ Let $\bar{x}_{i} x_{j}^{i} \rightarrow 0$ identically in $i$. Then $\overline{x x}_{i} \rightarrow 0$ if and only if $N(i)$ exists, so large that $j(i)>N(i)$ implies $\overline{x x}_{j(i)}^{i} \rightarrow 0$.

$(\gamma)$ There exist open sets $\dagger N_{1}, N_{2}, N_{3}, \cdots$, of which any open set is the sum of a subclass.

Employing Theorem 2 to replace $(\alpha)$ and $\left(\beta^{\prime \prime}\right)$ by equivalent axioms, and suppressing the hypothesis of local separability as being implied in the others (see Theorem 6), we obtain the following theorem.

THEOREM 11. Taken together with $(\gamma)$, the following convergence axioms determine which spaces ( $\Lambda$ ) satisfying (A) are PSM-spaces:

(B) If $z_{i} \rightarrow x$, and $z_{i} \rightarrow y$, then $x=y$.

(C) Let $x_{j}{ }^{i} \rightarrow x_{i}$ identically in $i$. Then $x_{i} \rightarrow x$ if and only if $N(i)$ exists, so large that $j(i)>N(i)$ implies $x_{j(i)}^{i} \rightarrow x$.

Finally, let $(\Im, K)$ be any topological space. Let us make the definition, $\left\{x_{k}\right\} \rightarrow x$ if and only if $\left\{y_{k}\right\} \subset\left\{x_{k}\right\}$-all points of the same infinite sequence being assumed distinct-implies $K\left(\left\{y_{k}\right\}\right) \supset x$. Then, since $\left(\mathrm{A}^{\prime}\right)$ and the new definition of convergence imply (A), we may state the following result.

THEOREM 12. The following convergence axioms determine which topological spaces are PSM-spaces:

$\left(\mathrm{A}^{\prime}\right) K(S) \supset x$ if and only if $\left\{x_{k}\right\} \subset S$ exists, such that $\left\{x_{k}\right\} \rightarrow x$.

(B) If $z_{i} \rightarrow x$, and $z_{i} \rightarrow y$, then $x=y$.

(C) Let $x_{j}^{i} \rightarrow x_{i}$ identically in $i$. Then $x_{i} \rightarrow x$ if and only if $N(i)$ exists, so large that $j(i)>N(i)$ implies $x_{j(i)}^{i} \rightarrow x$.

( $\gamma$ ) There exist open sets $\ddagger N_{1}, N_{2}, N_{3}, \cdots$, of which any open set is the sum of a subclass.

HARVARD UNIVERSITY

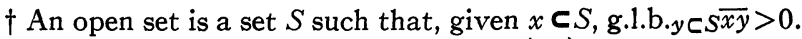

$\ddagger$ An open set is a set $S$ such that, if $\left\{x_{x}\right\} \subset \widetilde{S}-S$, and $x \subset S$, then $\left\{x_{x}\right\}$ does not $\rightarrow x$ 